\title{
Hahnemanns Theorie und Praxis und das moderne Erkenntnisproblem*
}

\author{
Josef M. Schmidt
}

Institut für Ethik, Geschichte und Theorie der Medizin, Ludwig-Maximilians-Universität München, Deutschland

\section{Schlüsselwörter}

Erkenntnistheorie · Gestalttheorie · Wirtschaftsgeschichte . Medizingeschichte $\cdot$ Homöopathie

\section{Zusammenfassung}

Anschauen, Wahrnehmen und Erkennen sind in der Medizin keine trivialen Vorgänge. Die moderne konventionelle Medizin hat sich diesbezüglich der Methode des klinischen Experiments und der Statistik sowie dem Ideal einer evidenzbasierten Medizin verschrieben, deren externe Evidenz letztlich auf «Erkenntnis ohne erkennendes Subjekt» im Sinne Karl Poppers beruht. Demgegenüber verfolgt die Homöopathie auch gestalttheoretische und kognitionsbasierte Ansätze wissenschaftlicher Erkenntnis im Sinne Karl Dunckers und Helmut Kienes. Unter Anerkennung der Urteils- und Erfahrungsfähigkeit des einzelnen Arztes ermöglicht dies die methodische Gewinnung interner Evidenz, die einer bloss metaanalytisch ausgerichteten Biomedizin verwehrt bleibt. Medizintheoretisch gesehen erweist sich der ganzheitliche Weg als dem Wesen des Arztberufs angemessener. Aus sozioökonomischen Gründen, die auf Vergesellschaftungsprozesse moderner Subjekte in der Denkform des Geldes und die Begründung der modernen Naturwissenschaft aus diesem Geiste zurückgehen, ist derzeit jedoch das biomedizinische Paradigma an der Macht. Indem Samuel Hahnemann die Homöopathie noch vor der zunehmenden Ökonomisierung der modernen Medizin im 19. Jahrhundert begründete, können er und seine Lehre heute als Leuchtturm einer exemplarischen, unverdorbenen Heilkunst dienen, die es trotz bzw. gerade wegen mannigfaltiger Anfechtungen wiederzubeleben gilt.

*Überarbeitete Version eines Vortrags, gehalten anlässlich der 162. Jahrestagung des Deutschen Zentralvereins homöopathischer Ärzte am 17.05.2012 im Konzerthaus Freiburg in Freiburg i.Br., Deutschland.

\section{Keywords \\ Theory of cognition - Gestalt theory · History of economics . History of medicine $\cdot$ Homeopathy}

\section{Summary}

Hahnemann's Theory and Practice and the Modern Problem of Cognition

The processes of viewing, perceiving, and recognizing are not trivial in medicine. In this regard, modern conventional medicine has committed itself to the method of clinical experiments and statistics as well as the ideal of an evidence-based medicine whose external evidence is ultimately based on 'cognition without cognitive subject' in the sense of Karl Popper. In contrast, homeopathy also pursues Gestalt-theoretical and cognition-based approaches of scientific cognition in the sense of Karl Duncker and Helmut Kiene. Acknowledging the ability to judge and learn on the part of the singular physician, this enables a methodological gathering of internal evidence, which a merely meta-analytically orientated biomedicine fails to accomplish. From the perspective of theory of medicine, the holistic way proves to be more appropriate to the essence of the physician's task. Due to socioeconomic reasons tracing back to socialization processes of modern subjects in the monetary form of thinking and the foundation of modern science out of that spirit, however, the biomedical paradigm actually is in power. Since Samuel Hahnemann had founded homeopathy still before the increasing economization of medicine in the nineteenth century, he and his doctrine may today serve as a lighthouse of an exemplary uncorrupt healing art presently besieged and waiting for its revival.

\section{KARGER \\ Fax +497614520714 Information@Karger.com} www.karger.com
Prof. Dr. med. Dr. phil. Josef M. Schmidt

Institut für Ethik, Geschichte und Theorie der Medizin

Ludwig-Maximilians-Universität München

Lessingstrasse 2, 80336 München, Deutschland

j.m.schmidt@lrz.uni-muenchen.de 


\section{Mots-clés}

Théorie de la connaissance - Théorie de la forme (gestaltisme) · Histoire de l'économie · Histoire de la médecine · Homéopathie

\section{Résumé}

Théorie et pratique de Hahnemann et le problème de la connaissance moderne

Regarder, percevoir et reconnaître ne constituent pas de processus triviaux en médecine. La médecine conventionelle moderne s'est, à cet égard, adonnée aux méthodes de l'expérimentation clinique et des statistiques ainsi qu'à l'idéal d'une médecine basée sur les faits (evidence-based medicine), dont les preuves externes reposent en fin de compte sur la «connaissance sans sujet connaissant» dans le sens de Karl Popper. L'homéopathie, au contraire, suit aussi des approches gestaltistes et basées sur la cognition de la connaissance scientifique dans le sens de Karl Duncker et Helmut Kiene. En admettant la capacité de discernement du médecin et sa capacité à acquérir de l'expérience, ceci permet d'obtenir méthodiquement des preuves internes, empêchant ainsi une biomédecine simplement méta-analytique. Du point de vue de la théorie de la médecine, cette méthode holistique se révèle plus conforme à la nature de la profession médicale. Pour des raisons socio-économiques, attribuées à des processus de socialisation des sujets modernes dans la logique de l'argent et à la fondation des sciences naturelles modernes qui sont issues de cet esprit, le paradigme biomédical est cependant prédominant actuellement. En fondant l'homéopathie avant l'économisation croissante de la médecine moderne au XIXe siècle, Samuel Hahnemann et sa doctrine peuvent aujourd'hui servir de guide d'une médecine non corrompue exemplaire qui s'avère valide malgré ou plutôt en raison des diverses critiques.

\section{Einführung}

Die Homöopathie befindet sich in einem eigenartigen Dilemma. Einerseits wird sie auf der ganzen Welt praktiziert und funktioniert offensichtlich recht gut, zur Freude unzählbarer Patienten und Therapeuten, andererseits behauptet die moderne konventionelle Wissenschaft, dass sie unwissenschaftlich, d.h. theoretisch im Rahmen heutiger Vorstellungen und Paradigmen von Arzneiwirkung nicht plausibel, sei und auch praktisch ihre vermeintliche Wirksamkeit nach derzeitigen methodischen Standards nicht belegen könne.

Hier fällt auf, dass eine Heilmethode, die vor etwa 200 Jahren entwickelt wurde [1], nach Kriterien, die 200 Jahre später die Medizin beherrschen, beurteilt wird. Für den kritischen Historiker, der um die Versuchung, Siegergeschichte zu schreiben, weiss, und erst recht für den Philosophen, der gegenüber der Vorstellung einer vermeintlich linearen Entwicklung von Gedanken im
Sinne einer Fortschrittsgeschichte Skepsis anmeldet, ist hier Vorsicht geboten [2]. Probleme der Wissenschaft, die auf den ersten Blick als Paradoxien erscheinen, sollten eigentlich eine intellektuelle Herausforderung sein, ihnen auf den Grund zu gehen. In der Tat lassen sich die meisten vermeintlichen Ungereimtheiten - wie zu zeigen sein wird - als Scheinprobleme auflösen, wenn man ihre Herkunft und Entstehungsbedingungen aufdeckt.

Im vorliegenden Fall soll mithilfe erkenntnistheoretischer, medizintheoretischer und sozioökonomischer Analysen, also mit legitimen Mitteln der Wissenschaft, einerseits gezeigt werden, dass das abschätzige Urteil vonseiten der konventionellen Medizin der Homöopathie nicht gerecht wird [3], andererseits aber auch, warum heute - mehr denn je - kaum Interesse daran besteht, diese Ungerechtigkeit aufzudecken, geschweige denn zu beseitigen.

\section{Der erkenntnistheoretische Rahmen}

Zunächst scheint es um ein rein erkenntnistheoretisches Problem zu gehen. Was Homöopathen «anschauen, wahrnehmen und erkennen» (so das Thema der 162. Jahrestagung des Deutschen Zentralvereins homöopathischer Ärzte (DZVhÄ) im Mai 2012), wird von der konventionellen Medizin grösstenteils nur deshalb nicht ernst genommen, weil deren Vertreter zu einer anderen Sichtweise der Dinge sozialisiert sind und daher ganz andere Aspekte der Wirklichkeit «anschauen, wahrnehmen und erkennen». Tatsächlich liegen hier zwei Paradigmensysteme vor (man könnte auch von kognitiven Paralleluniversen sprechen), von denen eines eben an der Macht und das andere in der Defensive ist. So unterschiedlich sie sind, so haben doch beide ihre je eigene Logik und Geschichte.

\section{Die randomisierte kontrollierte klinische Studie}

Die Art und Weise, wie heutige konventionelle Mediziner den Menschen und seine Krankheiten anschauen, wahrnehmen und erkennen, ist keineswegs selbstverständlich, sondern hat sich erst im Laufe der letzten vier Jahrhunderte in Europa herausgebildet. Diese Entwicklung hatte vor allem sozioökonomische Ursachen (die später noch erläutert werden). Aus erkenntnistheoretischer Sicht lassen sich, in einem ersten groben Überblick, vier Meilensteine benennen [4].

- Im 17. Jahrhundert hatte zunächst der englische Philosoph und Lordkanzler Francis Bacon (1561-1626), getragen von dem bereits in der Renaissance erwachten neuen Selbstbewusstsein der Reichen und Mächtigen, in seinem «Novum Organum Scientiarum» (1620) eine neue Methode der wissenschaftlichen Forschung vorgeschlagen, wonach nicht mehr das einfühlende 
Beobachten natürlicher Abläufe, sondern das Eingreifen in den Gang der Natur, also deren willentliche, absichtliche Manipulation, sichere Erkenntnisse liefern würde [5]. So wurde das Experiment zur massgeblichen Methode, um Kausalzusammenhänge zu entdecken, mit deren Hilfe es möglich sein sollte, die Natur nicht unbedingt besser zu verstehen, aber sie effektiver zu beherrschen.

- Im 18. Jahrhundert bezweifelte der schottische Philosoph und Ökonom David Hume (1711-1776) die Möglichkeit direkter Erkenntnis von Kausalität und behauptete stattdessen, dass wir immer nur die zeitliche Aufeinanderfolge von zwei Ereignissen (A und B) wahrnehmen, nie aber deren kausalen Zusammenhang. Wenn wir aber wiederholt sehen, dass auf A immer B folgt, nennen wir A die Ursache und B deren Wirkung. Obwohl zwar, ontologisch gesehen, jedes einzelne A die Ursache eines einzelnen B sein mag, können wir, erkenntnistheoretisch betrachtet, deren Kausalzusammenhang eigentlich nur dann erkennen bzw. vermuten, wenn wir sehr viele Abfolgen von $A$ und B beobachtet haben [6]. Abgesehen davon, dass wir auf diese Weise allenfalls Korrelationen zwischen A und B feststellen, wurde durch Hume die Wiederholung einer Beobachtung bzw. eines Experiments zum ausschlaggebenden Kriterium, um von einem «post hoc» zweier Ereignisse zu einer Aussage über deren "propter hoc» zu gelangen. Weil aber nie und von niemandem alle Fälle erfasst werden können, bleibe stets eine Unsicherheit zurück, dass die aufgrund der bisherigen Daten aufgestellte Hypothese mit der nächsten Beobachtung widerlegt werden könnte (Induktionsproblem).

- Im 19. Jahrhundert begründete der englische Philosoph und Ökonom John Stuart Mill (1806-1873) die sogenannte «Differenzmethode», mit der es möglich sein sollte, «auf dem Wege der unmittelbaren Erfahrung mit Gewissheit zu Ursachen (zu) gelangen». Hiernach müssen beim Vergleich zweier Fälle diese in allen Umständen gleich sein - bis auf den einen, den man untersuchen will. Weil aber in der Natur gleichartige Fälle sehr selten gegeben sind, müsse der Mensch diese Gleichheit künstlich herstellen. Bringt man zu einem der gleichartig gemachten Fälle einen Faktor A hinzu, zum anderen dagegen nicht, und tritt nur im ersten Fall eine Änderung B auf, im anderen dagegen nicht, so sei damit die Ursächlichkeit von A für B bewiesen [7]. Trotz aller Eingriffe des Untersuchers zur Herstellung gleicher Rahmenbedingungen folgt aber in der Medizin auf einen Faktor A nicht immer eine gleiche oder gleich starke Reaktion B, sodass hier trotz Anwendung der Differenzmethode eine einzelne Untersuchung meist nicht zu genügen scheint, sondern viele Patienten benötigt werden.
- Zur Behebung dieser Schwierigkeit hat im 20. Jahrhundert der englische Darwinist und Statistiker Ronald Aylmer Fisher (1890-1962) in seinem 1935 erschienenen Buch «The Design of Experiments» das Konzept der randomisierten Studie entworfen. Demnach muss der Forscher zweimal aktiv in das Experiment eingreifen. Noch vor seiner Applikation des Faktors A muss er zunächst eine Zufallskonstellation als Rahmenbedingung erzeugen, d.h., er stellt zwei zufallsverteilte Patientengruppen her. Wenn unter solchen Zufallsbedingungen der durchschnittliche Unterschied der Zielparameter mehr als zufällig, d.h. statistisch signifikant ist, dann soll diese Überzufälligkeit bedeuten, dass ein Ursache-WirkungZusammenhang vorliegt [8]. Das Kausalkriterium war fortan die statistisch auswertbare Überzufälligkeit. So wurde das Herzstück wissenschaftlichen Erkennens, nämlich das Erkennen von Kausalitätszusammenhängen, von der Statistik für die Statistik erobert.

In die klinische Forschung wurde die Methodologie der randomisierten Studien gleich nach dem Zweiten Weltkrieg übernommen. 1946 wurde die erste weltweit anerkannte randomisierte klinische Studie mit Streptomycin bei Lungentuberkulose durchgeführt [9]. Im selben Jahr wurde auf der "Cornell Conference on Therapy» in den USA als vordringlichster Forschungsgegenstand das Placebo festgelegt, und zwar - wie im Protokoll von 1946 nachzulesen ist -, um damit den offensichtlich enormen Erfolg der Homöopathie («the enormous success of homeopathy») zu erklären bzw. hinweg zu rationalisieren [10].

In diesem Zusammenhang gewann der 1955 im Journal of the American Medical Association erschienene Artikel «The Powerful Placebo» von Henry Beecher [11], nach dem angeblich 35\% der Patienten allein durch Placebo geheilt würden, eine bis zum heutigen Tag reichende Bedeutung. Wie aus einer medizinhistorischen Studie von Ted Kaptchuk hervorgeht, war das Placebo-Argument damals die Eintrittskarte in die Epoche der verblindeten randomisierten Studien (randomized controlled trial (RCT)). Es diente konkret dazu, angesichts der damit beschworenen Bedrohung der wissenschaftlichen Wirksamkeitsbeurteilung die medizinische Profession zu überreden, fortan die placebokontrollierte randomisierte Studie als neuen Goldstandard zu akzeptieren [12].

In den 1960er-Jahren prägte die Methodenlehre der randomisierten Studie bereits die Arzneimittelgesetzgebung der USA und in den 1970er-Jahren auch jene Mitteleuropas. Die 1980er-Jahre brachten, ermöglicht durch die neuen elektronischen Medien (Computer), die zusammenfassende Auswertung klinischer Studien, d.h. die Metaanalysen, und in den 1990er-Jahren kam es zu einer zunehmenden internationalen Standardisierung 
von Prüfrichtlinien und global vernetzten Auswertungen [13].

So faszinierend es sein mag, das Problem wissenschaftlicher Erkenntnis mithilfe der Mathematik bzw. Statistik auch nur teilweise oder annähernd in den Griff zu bekommen, wie es unter anderen bereits dem französischen Mathematiker Pierre-Simon Laplace (1749-1827) vorschwebte, so ist doch keineswegs zu übersehen, dass es ausser der statistischen Erfassung und Auswertung von Studiendaten auch andere Möglichkeiten gibt und immer schon gab, um reale Phänomene und kausale Zusammenhänge auf wissenschaftliche Weise wahrzunehmen und zu erkennen.

\section{Theorie der Gestaltwahrnehmung}

Ein grundsätzlich anderes Konzept von Anschauen, Wahrnehmen und Erkennen wurde z.B. von dem deutschjüdischen Gestalttheoretiker Karl Duncker (1903-1940) in seinem 1935 erschienenen Buch «Zur Psychologie des produktiven Denkens» entwickelt, das auf Vorarbeiten von Christian von Ehrenfels, Max Wertheimer, Wolfgang Köhler, Kurt Koffka, Kurt Lewin und Wolfgang Metzger aufbaut $[4,14]$.

Ausgangspunkt für eine eigenständige Theoriebildung in der Gestaltpsychologie war die Beobachtung, dass beim gewöhnlichen Wahrnehmen einheitliche Zusammenhänge auch unabhängig von den Teilen, aus denen sie bestehen, erkannt werden, etwa eine Melodie oder ein Akkord als solche(r) unabhängig von der Tonhöhe oder eine Figur als solche unabhängig von ihrer Grösse oder Farbe usw. Die Wahrnehmung einer solchen «Gestalt» ist zwar empiristisch gerade nicht erklärbar, beruht aber dennoch auf konkreten Gestaltgesetzen, wodurch etwas als zusammengefügt wahrgenommen wird, oder auf Ganzheitsbedingungen, wie den Faktoren der Nähe, der Gleichheit, der guten Gestalt, der guten Kurve, der Geschlossenheit oder des gemeinsamen Schicksals. So gibt es eine Raumgestalt, Zeitgestalt, Bewegungsgestalt, Prozessgestalt und eben auch einen «Gestaltfaktor phänomenaler Kausalität».

Ein Kausalzusammenhang ist z.B. dann einsichtig, wenn Zeit und Ort der Ursache unmittelbar in Zeit und Ort der Wirkung anschaulich eingehen, wenn also etwa der Rhythmus eines Klopfgeräuschs dem Rhythmus der Klopfbewegung entspricht oder wenn eine Spur (z.B. im Schnee) dem sie hinterlassenden Gegenstand gleicht. Formentsprechungen liegen vor, wenn etwa die Nässe des Regens in die Nässe der Strasse übergeht oder die Farbe des Lichts auf den beleuchteten Gegenstand oder die Bewegung des Stossenden in die Bewegung des Gestossenen. So sind Ursache und Wirkung nicht nur durch räumliche und zeitliche Koinzidenz, sondern auch bezüglich des Inhalts einsichtig zueinander: «Eigenschaften der Form, des Charakters, der Richtung, des Materials usw. gehen unmittelbar anschaulich aus der Ursache in die Wirkung ein» [14].

\section{Kognitionsbasierte Medizin}

Die Einsichtigkeit und Wahrnehmbarkeit solcher inhaltlicher Entsprechungen baute Helmut Kiene, anknüpfend an seinen Lehrer Gerhard Kienle (1923-1983), für die Medizin zu einer eigenen Erkenntnismethode aus, der sogenannten «Komplementären Methodenlehre der klinischen Forschung» [4]. Demnach gibt es drei Haupttypen des Kausalerkennens, in folgender Abstufung:

1. Die einfachste und direkteste Form eines sicheren Kausalerkennens ist die sogenannte ideelle Methode, das unmittelbare Erfassen einer Kausalgestalt, etwa die Ursächlichkeit von Reposition, Retention und Ruhigstellung eines Knochenbruchs für ein orthogrades kallusfreies Zusammenwachsen desselben oder die Ursächlichkeit einer Tracheotomie für die Behebung akuter Atemnot bei einer Obstruktion des Rachenraums. Wo immer dieses unmittelbare Erkennen möglich ist, bedarf es keines experimentell-vergleichenden Ansatzes, sondern es genügt die Wahrnehmung einer in sich geschlossenen Prozessgestalt.

2. Etwas komplizierter ist bereits die abbildende Methode, das Erfassen einer Kausalgestalt auf der Grundlage einer abbildenden Korrespondenz, bei der die Gestaltübereinstimmung zwischen einer gezielt vorgenommenen (und variierten) Handlung und ihrer Wirkung wahrgenommen wird, etwa wenn sich die Bewegung der Computermaus direkt aufdie Bewegung des Cursors auf dem Monitor abbildet oder sich eine Variation der Arzneimitteldosis proportional auf den Gesundheitszustand des Patienten auswirkt. Hier ist methodisch eine intentionale Aktivität erforderlich, damit ein erkennbarer Kausalzusammenhang hergestellt werden kann.

3. Bei der statistischen Methode des Kausalerkennens handelt es sich - so gesehen - um einen Sonderfall der abbildenden Methode, denn hier wird auch eine Gestalt, allerdings nur eine rudimentäre, nämlich ein blosser Unterschied, abgebildet. Die Fragestellung lautet hier: Bildet sich der Unterschied der Behandlung (Behandlung X vs. Y) als ein statistisch signifikanter Unterschied der Zielparameter ab (Ergebnis x vs. $y$ )? Diese Reduktion auf einen Zahlenwert lässt sich nur durch die Durchführung einer randomisierten Studie kompensieren, die aber einen doppelten Eingriff des Forschers verlangt: die Erzeugung gleicher Gruppen (durch Randomisation) und deren unterschiedliche Behandlung (mittels Placebokontrolle).

Dargestellt in dieser Rangordnung, erscheint die gestalttheoretische Methode des Kausalerkennens freilich als die Methode der Wahl, da sie die vielen Nachteile von 
randomisierten Studien - von der ethisch bedenklichen Belastung grosser Patientenkollektive über den hohen Kostenaufwand bis zur immanenten Tendenz zu falsch negativen Ergebnissen - vermeidet und stattdessen flexibel und effizient Kausalitätserkenntnis am individuellen Einzelfall ermöglicht. Über das Erkennen rein kausaler Zusammenhänge hinaus werden mit der gestalttheoretischen Methode gleichzeitig auch noch Wesensbeziehungen etwa zwischen Mensch und Arzneisubstanz erfasst, komplexe Muster bestimmter Symptomatologien wahrgenommen oder spezielle Einblicke in Funktionsoder Prozessgestalten realisiert.

Einschränkend ist hier allerdings zu bemerken, dass in der Medizin die hohe Isomorphie zwischen Ursache und Wirkung, wie sie in den Beispielen von Duncker und Kiene vorausgesetzt wird, nicht immer gegeben ist. Im Bereich des Organischen sind äussere Einwirkungen oft weniger als Ursachen im strengen Sinne, sondern vielmehr als Bedingungen aufzufassen, auf die der Organismus aktiv und je nach seinen Möglichkeiten und seiner Gestimmtheit «antwortet» $[15,16]$. In diesem Fall kann von der Symptomatik des Patienten nicht direkt gestalttheoretisch auf eindeutige Ursachen und Therapien geschlossen werden, sondern das Verhalten des Patienten ist - unter Berücksichtigung seiner individuellen Umwelt und im empathischen Nachvollzug seiner Bedeutungsprozesse - hermeneutisch und semiotisch zu verstehen [17]. Da dieser Ansatz bereits an anderer Stelle ausführlich dargestellt ist, soll er hier nicht weiter vertieft werden [18].

\section{Evidenzbasierte Medizin}

Um die Brisanz, die Bedeutung und die Konsequenzen der ganz anderen Art des Anschauens, Wahrnehmens und Erkennens, wie sie von der Gestalttheorie vorgeschlagen wird, deutlich zu machen, sei kurz auf ihr komplettes Gegenteil eingegangen, nämlich auf das Wissenschaftskonzept des österreichisch-jüdischen Rationalisten Karl Popper (1902-1994), das bis heute in der konventionellen Medizin als grundlegend anerkannt ist. Was Popper unter wissenschaftlicher Erkenntnis versteht, schreibt er in seinem Buch "Objektive Erkenntnis»: «Wissenschaftliche Erkenntnis ist nicht Erkenntnis im gewöhnlichen Sinne von «Ich kenne»! Diese Erkenntnis gehört zur Welt des persönlichen Subjekts ... Erkenntnis im objektiven Sinne ist Erkenntnis ohne einen Erkennenden: Es ist Erkenntnis ohne erkennendes Subjekt» [19]. Als Antwort auf David Humes Induktionsproblem versagt Popper der Wissenschaft jeden Anspruch auf Wahrheit. Sie könne vielmehr immer nur Hypothesen formulieren, die nie bewiesen, sondern allenfalls durch Falsifikation verworfen werden können. Wie der Wissenschaftler aber überhaupt zu positiven Einsichten, und seien sie auch nur Hypothesen, kommen soll, darüber schweigt er sich aus.
In seinem 1935 erschienenen Buch «Logik der Forschung» schrieb Popper: "Wir wollen ... die Aufgabe der Erkenntnistheorie ... derart bestimmen, dass sie lediglich die Methoden der systematischen Überprüfung zu untersuchen hat.» Zur Entstehung von Erkenntnissen meinte er: «Jede Entdeckung enthalte ein irrationales Element), sei eine «schöpferische Intuition»» [20]. Daher gelte: «Keine Erkenntnistheorie sollte versuchen zu zeigen, warum Erklärungen gelingen» [19]. Wer sich auf dieses Programm einlässt, und die konventionelle Medizin tut dies mit der Anerkennung Poppers, hat jede Hoffnung aufgegeben, jemals etwas Wahres erkennen zu können. Er spricht diesen Anspruch damit auch allen anderen ab (etwa Hahnemann und der Homöopathie) und beschränkt seine wissenschaftliche Kompetenz darauf, auf vermeintlich objektive (d.h. subjektlose) Weise Hypothesen anderer zu widerlegen.

Noch deutlicher wird die Andersartigkeit und Eigenständigkeit der von Helmut Kiene vorgeschlagenen gestalttheoretischen Methode des Kausalerkennens, wenn man sie - als sogenannte «Cognition-based Medicine» [4] - der «Evidence-based Medicine» gegenüberstellt. Laut einem ihrer geistigen Väter, dem kanadischen Biostatistiker David Sackett ( ${ }^{*} 1934$ ), soll «die Praxis der Evidenzbasierten Medizin ... die Integration individueller klinischer Expertise mit der externen Evidenz aus systematischer Forschung» bedeuten [21]. Wie selbstverständlich wird dabei unterstellt, dass der einzelne Arzt - bei aller ihm zugestandenen «individuellen klinischen Expertise» - ausserstande sei, für sich selbst wissenschaftliche Evidenz aufzubauen (etwa durch Einzelfallbeobachtungen), sondern auf sogenannte externe Evidenz (gemeint sind randomisierte Studien) angewiesen sei.

Erklärtes Ziel der randomisierten Studie war es aber gerade, das menschliche Urteil auszuschalten, wie bei dem englischen Biostatistiker Stuart Pocock in seinem Buch «Clinical Trials» nachzulesen ist: «to guard against any use of judgment» [22]! Anstatt die im Rahmen der eigenen Ausbildung erworbenen persönlichen Anschauungen, Wahrnehmungen und Erkenntnisse einbringen zu können, wird der Arzt auf diese subjektausschaltende Weise quasi zum Erfüllungsgehilfen der Ergebnisse klinischer Studien - jener Maschinerien, die von subjektiven Erkenntnisleistungen ohnehin unabhängig sind.

\section{Auswirkungen auf die Homöopathie}

Demgegenüber geht es einer kognitionsbasierten Medizin darum, eine andere (möglicherweise zusätzliche) Art von interner wissenschaftlicher Evidenz aufzubauen, die auf der individuellen Urteils- und Erfahrungsfähigkeit des einzelnen Arztes beruht, auf der Grundlage abbildender Korrespondenz sowie der Erfassung von funktionellen, pathogenetischen und therapeutischen Kausalgestalten. Mit der gezielten Förderung einer pro- 
fessionellen Ausbildung der individuellen Erfahrungsbildung des Arztes wäre zudem auch eine höhere Wertschätzung der medizinischen Vergangenheit sowie der Autoritäten der Tradition verbunden.

Unter diesen ganz anderen Vorzeichen liessen sich weder Hahnemann und seine Nachfolger noch die Homöopathie als Ganze ignorieren und verwerfen, wie dies vom Paradigma der Evidence-based Medicine aus gegenwärtig geschieht. Im Gegenteil, anhand der Schriften und Aufzeichnungen von Hahnemann und seiner Schüler lässt sich heute noch lernen, wie methodische Wesenserkenntnis am Einzelfall funktioniert, ohne randomisierte Doppelblindstudien oder Ähnlichem $[23,24]$.

\section{Der medizintheoretische Hintergrund}

Eine weitere Erwiderung auf den Vorwurf, Hahnemanns Theorie und Praxis sei mit der heutigen Art des Wissenserwerbs und dessen Anwendung in der Medizin unvereinbar, ist von der neueren Medizintheorie her möglich.

\section{Medizin als Handlungswissenschaft}

Nachdem sich die moderne Medizin, unter dem zunehmenden Druck des sichtbaren Fortschritts der aufstrebenden Naturwissenschaften, im 19. Jahrhundert dazu entschlossen hatte, sich fortan als angewandte Naturwissenschaft $\mathrm{zu}$ verstehen und zu präsentieren, gibt es seit einigen Jahrzehnten - in der Theorie der Medizin - wieder dazu gegenläufige Strömungen. Aufbauend auf den Ausführungen des deutschen Medizinphilosophen Wolfgang Wieland ${ }^{*}$ 1933) [25] hebt unter anderen der deutsche Medizintheoretiker Urban Wiesing ${ }^{*}$ 1958) hervor, dass die Medizin primär keine erkennende, sondern stets nur eine praktische Wissenschaft sui generis sein könne bzw. müsse [26].

Grundlage der Medizin könne demnach nie ein abstraktes Erkenntnisinteresse des Arztes sein, sondern immer nur der konkrete Auftrag des Patienten an den Arzt, zum Nutzen und Besten des Patienten tätig zu werden. Als Handlungswissenschaft unterliege die Medizin aber ganz anderen Kategorien als z.B. die Naturwissenschaften, bei denen die künftige Anwendung von Erkenntnissen sekundär oder kontingent sei, jedenfalls nicht zu ihrem Wesen gehöre. Der entscheidende Unterschied zwischen dem Wesen der Medizin und dem der Naturwissenschaften liesse sich bei dieser Argumentation letztlich auf den Unterschied von Handeln und Wissen zurückführen.

Nach einer Zusammenfassung von Wiesing [27], die auf Wieland [25] zurückgeht, sind an Handeln und Wissen ganz verschiedene Anforderungen zu stellen:
- Handeln muss legitimiert werden, während Wissen verifiziert werden muss.

- Handeln ist verbindlich zu normieren. Diese Forderung lässt sich an Wissen nicht sinnvollerweise herantragen, weil Wissen nur wahr oder falsch sein kann.

- Während eine Handlung stets für eine Person verbindlich ist, kann Wissen als solches wahr oder falsch sein.

- Handeln ist entweder erlaubt, geboten oder verboten. Wissen dagegen, vor allem in den Biowissenschaften, ist meist statistischer Natur.

- Handeln ist prinzipiell irreversibel (lediglich die Folgen können reversibel sein). Das genaue Gegenteil gilt für das Wissen.

- Während praktisches Handeln meist nicht exakt zu vollziehen ist, unterliegt das Wissen der Anforderung auf Exaktheit.

- Handeln ist nicht zu einem bestimmten Teil zu vollziehen: «Man kann Handlungen nicht lediglich mit einer gewissen Wahrscheinlichkeit realisieren» [28]. Eine These hingegen, selbst wenn sie eine statistische Aussage enthält, kann man mit einer bestimmten Wahrscheinlichkeit vertreten.

Bereits anhand dieser Übersicht über die grundsätzliche Verschiedenheit der Kategorien Wissen und Handeln wird das Problem deutlich, das sich ergibt, wenn man - wie in der Medizin unweigerlich - versucht, das Produkt reiner Erkenntniswissenschaften, nämlich abstraktes Wissen, mit der Verpflichtung des Arztes zum konkreten Handeln im einzelnen Fall in Verbindung zu bringen [29].

\section{Medizin als Heilkunst}

Wie es in der Medizin wissenschaftlich begründetes Handeln geben könne, kann nun aber gerade nicht Forschungsgegenstand einer reinen Erkenntniswissenschaft, etwa einer Naturwissenschaft, sein. "Wie Wissen in Handeln umzusetzen sei, eben das lehrte die ärztliche Kunst. Mit der Eliminierung der ars medica aus der wissenschaftlichen Medizin war auch der Ort verlorengegangen, an dem in Forschung und Lehre dieser zentrale Akt ärztlicher Handlung die ihm gebührende Aufmerksamkeit hätte finden können» [28].

Wenn also im Rahmen einer konsequenten Ausrichtung der modernen konventionellen Medizin auf die Richtlinien der Evidence-based Medicine die Ebene der erkenntnistheoretischen Grundlagenreflexion übersprungen und durch Erkenntnistechnologie ersetzt wird, so mag dies zwar im ersten Moment wie eine Arbeitserleichterung erscheinen. Einer Heilkunst im klassischen Sinne wie auch im neueren medizintheoretischen Verständnis dürfte man damit aber nicht näher gekommen sein.

Demgegenüber begriff sich Hahnemann stets und bewusst als «Heilkünstler», d.h. als einen, dem es - wie 
seinem Vorbild Hippokrates, in dessen Tradition er sich sah - primär um die Erfüllung seines praktischen Auftrags seinen erkrankten Mitmenschen gegenüber ging. Dementsprechend interessierte ihn primär nicht jedes theoretisch erhebbare, sondern nur praktisch verwertbares Wissen [30, 31]. Auf diese Weise entstand eine Heilkunde, deren praktischer Wert den Test von 200 Jahren offensichtlich überstanden hat - was man von den Ergebnissen heutiger randomisierter Doppelblindstudien eher nicht erwarten wird, zumal bereits heute klar ist, dass die meisten publizierten Ergebnisse ohnehin falsch sind [32].

\section{Die sozioökonomischen Ursachen}

Die spannendste Frage zu dem Thema sei zum Schluss noch in Grundzügen angerissen und behandelt. Wie kommt es, dass, obwohl die Homöopathie - erkenntnistheoretisch und medizintheoretisch betrachtet - offenbar über beste Voraussetzungen verfügt, dem Ideal einer wissenschaftlichen und wohltätigen Heilkunst nahezukommen und von allen begrüsst und gefördert zu werden, ihr aber dennoch vonseiten der modernen konventionellen Medizin die Anerkennung versagt wird? Anders gefragt: Wieso hat nicht die Homöopathie, sondern die moderne konventionelle Medizin die Deutungshoheit über die Frage der Wissenschaftlichkeit in der Medizin und - möglicherweise damit zusammenhängend - eine sozioökonomische Basis, die den Marktanteil der Homöopathie um mindestens das Tausendfache übertrifft (es geht hier um die Grössenordnung von Billionen vs. Milliarden US Dollar Jahresumsatz weltweit)?

Offenbar werden hier die theoretischen Ergebnisse erkenntnistheoretischer und medizintheoretischer Analysen von einer ganz anderen Realität eines Besseren belehrt - die sich aber gleichwohl wissenschaftlich fassen lässt, eben durch die Geschichts- und Wirtschaftswissenschaften.

\section{Erkenntnis und Interesse}

Zur Einstimmung in diesen anderen Themenkreis ist es nützlich, sich klar zu machen, dass auch die Erkenntnistheorie, als typische Erkenntniswissenschaft, zwar rational danach fragt und darauf reflektiert, was "wir» erkennen können und was nicht, dabei aber die dahinterstehende Absicht, etwas Bestimmtes erkennen zu wollen, unreflektiert lässt. Da auch Erkenntnistheoretiker meist keine freien Geister, sondern Menschen sind, die als solche dem jeweiligen Zeitgeist und mit diesem sozioökonomischen Rahmenbedingungen und Machtkonstellationen unterworfen sind, sind die Wahrheiten und Einsichten, die sie hervorbringen, keineswegs absolut, sondern immer auch auf die Interessenlage der jeweiligen Epoche zu beziehen.
So könnte eine Geschichte der Erkenntnistheorie zeigen, wie ein und dieselbe Frage nach den Möglichkeiten und Grenzen unserer Erkenntnis etwa bei Platon noch darauf abzielte, die Ideen des Guten, Schönen, Gerechten usw. zu schauen, im Mittelalter darauf, Gottes Willen zu erkennen und gemäss der von ihm vorgesehenen Ordnung zu leben, in der Neuzeit darauf, die eigenen Rechte als Individuum $\mathrm{zu}$ entdecken und gleichzeitig die Schwachstellen der nun als hinderlich erkannten Religion blosszustellen, in der Moderne darauf, einer säkularisierten Bürgerschaft zu Besitz, Einfluss und Wohlstand zu verhelfen, und in der Postmoderne darauf, durch Dekonstruktion von Normen und Autoritäten individuelle Freiräume für jeden und alles zu erstreiten usw.

Die Frage, wie es dazu kam, dass sich der erkenntnistheoretische und medizintheoretische Diskurs der letzten Jahrhunderte dahin entwickelt hat, dass die Medizin letztlich als angewandte Naturwissenschaft und die randomisierte klinische Studie als Standardmethode des Wirksamkeitsnachweises angesehen wurde, ist also an die Soziologie und Ökonomik zu verweisen und, sofern überhaupt, dort zu lösen.

\section{Geld und Gesellschaft}

Als Propädeutik der Soziologie gilt es dabei zu bedenken, dass Menschen soziale Wesen sind, die sich zusammenschliessen und Gesellschaften bilden, d.h. sich vergesellschaften, und zwar auf spezifische Weise. Da eine Gesellschaft nicht eine Aggregation materieller Dinge ist, sondern das Ergebnis mentaler Bedeutungsprozesse, die von den einzelnen Individuen kollektiv vollzogen werden, hängt das, was sich bei dem permanenten Vergesellschaftungsprozess an Gesellschaft ergibt, ganz davon ab, auf welche Weise bzw. in welcher Form die Vergesellschaftung geschieht.

So lässt sich z.B. die Sprache als ein soziales Phänomen auffassen, das nicht für sich allein, losgelöst von ihren Sprechern existieren kann, sondern davon abhängt, durch ständiges Sprechen (bzw. Lesen und Schreiben) am Leben erhalten zu werden. Eine Sprache kann sich im Laufe der Zeit - je nachdem, wer mit welchem Einfluss in ihr mitspricht - zwar verändern. In den meisten Fällen ist es jedoch so, dass das Kleinkind in seine jeweilige Muttersprache, die immer schon vor ihm da ist, hineinwächst und zu ihrem Sprecher wird, d.h. sie weiter reproduziert, ohne eine Alternative zu haben [33].

Wie die Sprache, so findet jedes neue Mitglied einer Gesellschaft auch immer schon spezifische Denk- oder Rechenstrukturen vor, über deren Reproduktion sich die Gesellschaft als solche erhält. Die heute weltweit dominierende Form der Konstitution und Reproduktion von Gesellschaften ist die Vergesellschaftung in der Form des Geldes [34]. Der damit unmittelbar gegebene Einfluss des Geldes als Denkform im Vergesellschaftungsprozess 
der Staaten lässt sich durch die ganze Kulturgeschichte der Welt bis zur ersten Prägung von Geldmünzen in Lydien (Kleinasien) im siebten vorchristlichen Jahrhundert zurückverfolgen $[35,36]$. König Krösus von Lydien (590-541 v. Chr.) war im 6. Jahrhundert v. Chr. einer der Ersten, der mit frisch geprägtem Münzgeld Eroberungskriege gewann und sehr reich wurde - was ihm letztlich aber zum Verhängnis wurde.

Die klassischen Philosophen der Antike, Platon (428348 v. Chr.) und Aristoteles (384-322 v. Chr.), kritisierten bereits im 4. Jahrhundert v. Chr. das Um-sich-Greifen des allgemeinen Trachtens ihrer Zeitgenossen nach Gelderwerb, hatten aber durch ihre Ausrichtung auf Schönheit und Harmonie ein hinreichendes Gegengewicht, um nicht selbst in den Sog zu geraten. Aristoteles unterschied zwischen Oikonomía, der Haushaltsführung, die auch den Tausch von Waren gegen Geld und umgekehrt umfasste, und Chremastiké, der Kunst des Gelderwerbs um des Gelderwerbs willen. Letztere lehnte er ab, weil sie der Natur des Menschen widerspreche [37].

Im Mittelalter war der Geldverkehr aufgrund der überwiegenden Subsistenzwirtschaft wieder sehr begrenzt. Gleichwohl hat das Gelddenken als Denkform erneut in der Scholastik Eingang in theologische Gedanken und politische Organisationsformen gefunden (etwa bei Roger Bacon (1214-1292) und Wilhelm von Ockham (1288-1347)).

In der Renaissance hatte die durch die Aushöhlung des Zinsverbots ermöglichte Ausbreitung des Bankenwesens zur Folge, dass mit der neuen Verbreitung und Bedeutung des Geldes (als Denkform) ein neues Denken und damit gleichsam ein neuer Mensch geschaffen wurde. Ökonomisch motivierte Entdeckungsreisen rund um die Welt, die Annexion und Ausplünderung Amerikas, Kolonisierung, Sklavenhandel, Beutezüge von Piraten im Dienste Ihrer Majestät, etwa durch Francis Drake (1540-1596) in der Karibik, usw. mögen als Stichworte für den von der Gier nach Geld bestimmten neuen Zeitgeist fungieren, so wie Namen wie Niccolò Machiavelli (1469-1527) und Thomas Hobbes (1588-1679) für die ihn rechtfertigende Philosophie [38, 39].

\section{Moderne Naturwissenschaft}

In eben diese Zeit fällt die Geburt der Methode der modernen Naturwissenschaften aus dem Geiste der Ökonomie, wie sie von Francis Bacon (1561-1626) und Galileo Galilei (1564-1642) vollzogen wurde [40]. Naturwissenschaften wurden deswegen gerade zu dieser Zeit (und nicht etwa 500 Jahre früher) interessant, weil mit ihrer Hilfe Erfindungen gemacht werden konnten, mit denen liquide Unternehmer und Anleger sich die Vermehrung ihres dafür eingesetzten Geldes versprachen. Die Philosophie von René Descartes (1596-1650) wiederum ist Ausdruck der sich im 17. Jahrhundert vollziehenden
Konstitution des modernen vermeintlich autonomen Subjekts, das, indem es ganz aus den neuen ökonomischen Rahmenbedingungen hervorging, auch Geldsubjekt genannt werden kann [34].

Da der Begriff «ratio» von der kaufmännischen Rechnungslegung im alten Rom stammt, lässt sich das Zeitalter des nun anhebenden Rationalismus am besten als vom Vergesellschaftungsprozess in Form des Gelddenkens geprägt begreifen. Wer sich fortan nicht mehr auf Moral und Familienbande verlassen konnte und in seiner Existenz ganz von den Bewegungen des Geldes abhängig wurde, die er nur machtlos und ratlos in Form von schwankenden Aktienkursen verfolgen konnte, hatte eben Grund, an allem zu zweifeln (ausser an seinem Zweifel; wie Descartes). Oder er glaubte, dass wir ohne ständige Sinnesreize von aussen innerlich völlig leer wären (tabula rasa; John Locke (1632-1704)) oder dass wir nur noch zeitliche Aufeinanderfolgen von Ereignissen registrieren können, aber zu keiner Wesenserkenntnis mehr fähig seien (David Hume).

Mit David Hume, der, wie John Locke, nicht nur Philosoph, sondern auch Ökonom war und als solcher unter anderem Erkenntnistheorie trieb, schliesst sich der Kreis zur eingangs thematisierten Entwicklung der Methodik der konventionellen Medizin in der Moderne. Zunehmende Ökonomisierung, Industrialisierung und Monetarisierung aller Lebensbereiche sorgten in den letzten beiden Jahrhunderten dafür, dass es heute so gut wie keinen Kultur- oder Wissenschaftsbereich mehr gibt, der nicht vom Geld bzw. von den Märkten beherrscht wäre. Dies gilt auch für den modernen konventionellen Medizinbetrieb und die dort vorherrschenden Methoden und Prioritäten [41, 42]. Statistisch gesehen dürfte der Zusammenhang zwischen der wachsenden Herrschaft des Geldes im 19. und 20. Jahrhundert und der gleichzeitigen Entwicklung methodischer Prinzipien wie Objektivierbarkeit, Materialisierbarkeit, Quantifizierbarkeit, Mathematisierbarkeit, Standardisierbarkeit, Reproduzierbarkeit usw. eindeutig überzufällig, und damit signifikant, sein. Gestalttheoretisch ist er jedenfalls bei näherer Betrachtung ohne Weiteres wahrzunehmen und zu erkennen.

\section{Homöopathie}

Hahnemanns Theorie und Praxis nimmt in dieser Geschichte eine Sonderstellung ein. Zum einen lebte er noch in der Zeit, als die Industrialisierung und Beschleunigung des Lebens zumindest in deutschen Kleinstädten erst langsam anhob. Sein Wirken und seine Philosophie kann demnach als noch relativ frei von dem erst nach seinem Tod sich Bahn brechenden Trend zur Vernaturwissenschaftlichung und Kommerzialisierung der Medizin betrachtet werden. Zum anderen war er auch als sittlich aussergewöhnlich gefestigte Persönlichkeit imstande, 
mögliche Versuchungen einer Einflussnahme ökonomischer Anreize auf seine medizinische Theorie und Praxis abzuweisen. Immerhin war er Freimaurer und damit einem aufrichtigen und rechtschaffenen Leben zum Wohle seiner Menschenbrüder verpflichtet [1].

Diese Ausnahmestellung Hahnemanns, sowohl historisch als auch persönlich, ist aber für die Homöopathie und ihre Zukunft kaum überzubewerten. So wie in der Praxis die Resultate der Arzneimittelprüfungen, die er vor 200 Jahren durchgeführt hat, auch heute noch gültig sind, musste auch seine Theorie aufgrund ihrer Solidität und Schlichtheit bis dato kaum ergänzt oder überarbeitet werden. Im Gegensatz zu seinen heutigen vermeintlichen Weiterentwicklern $[43,44]$ war Hahnemann noch frei von dem Innovationsdruck, der in modernen kapitalistischen Staaten herrscht, ebenso wie vom pausenlosen Kampf um Marktanteile, der sich inzwischen im gesamten und somit auch im alternativen Bereich der Medizin abspielt. Zu seinem Glück konnte Hahnemann schliesslich eine Form von Praxis finden, in der er sich als freier Mann ganz seiner ärztlichen Aufgabe widmen und sich und seiner Familie gleichsam nebenbei den Lebensunterhalt verdienen konnte [45], ohne durch internationale Leitlinien, obligatorische Fortbildungsveranstaltungen, Qualitätszirkel, Konsensuskonferenzen oder Ähnliches gegängelt und manipuliert zu werden. Das Vermächtnis, das er unter diesen heute nicht mehr gegebenen Umständen der Nachwelt hinterliess [46], kann sich jedenfalls sehen lassen, auch und gerade vonseiten der modernen konventionellen Medizin - als Anregung, ihre Konzepte von «Anschauen, Wahrnehmen und Erkennen» neu zu überdenken.

\section{Schlussfolgerungen und Ausblick}

Unter den verschiedenen theoretisch möglichen Formen von Erkenntnis hat sich die konventionelle Medizin im 19. Jahrhundert für den Weg der vermeintlich objektiven, dabei aber weitgehend subjektlosen Erkenntnisgewinnung durch Vermessung und Manipulation des (abstrakten) Patienten entschieden. Diese Entwicklung erscheint vor dem Hintergrund der Wirtschafts- und Sozialgeschichte keineswegs kontingent, sondern so kon- sistent und kohärent, dass sich die These nahelegt, das eigentliche Subjekt der modernen westlichen Gesundheitssysteme seien weniger menschliche Individuen als vielmehr die Denkform des Geldes, in der die Vergesellschaftung und damit auch die Organisation der Medizin in Forschung, Lehre und Praxis geschieht. So erfreulich die vielfachen karitativen Impulse wohlwollender Helfer und Helferinnen sind, so stehen sie doch in der Regel einer Übermacht systemischer ökonomischer Zwänge gegenüber, die im Konfliktfall wie selbstverständlich den Ausschlag geben.

Demgegenüber basiert die Homöopathie seit jeher auf einem Menschenbild, das - neben dem kausalanalytischen Ansatz [47] - auch Raum lässt für hermeneutische, semiotische und gestalttherapeutische Weisen des Erkennens und somit ein ganzheitliches Verstehen und Behandeln jedes einzelnen Patienten erlaubt [48]. Aus einer sozial-, ökonomie- und wissenschaftshistorischen Perspektive stellt die persistierende Existenz dieser Form von Praxis allerdings einen Anachronismus, eine Anomalie, ja ein Skandalon dar - insofern sich hier etwas gegen die nivellierende Macht des Mainstreams durchhält, was sich zudem einem einfachen Begreifen mit modernen reduktionistischen Kriterien entzieht.

Unter dem Druck der sogenannten Evidenz-basierten Medizin neigen Homöopathen - wie zu Zeiten der Dominanz der naturwissenschaftlich-kritischen Richtung innerhalb der Homöopathie [44, 49] - heute wieder dazu, die Homöopathie durch Reduktion ihrer Methode und ihres Erkenntnisrahmens auf ein von der konventionellen Medizin anerkanntes Format bis zur Unkenntlichkeit und Unwirksamkeit zu verstümmeln. Sinnvoller und nachhaltiger wäre es dagegen, die Eigentümlichkeit und Sperrigkeit der Homöopathie gegenüber dem Zeitgeist der Ökonomisierung, Standardisierung und Reproduzierbarkeit eher als ererbten Schatz zu würdigen und zu bewahren und sich in methodischen und ethischen Fragen im Zweifelsfall an Hahnemann als Leuchtturm einer unverdorbeneren Zeit zu halten.

\section{Disclosure Statement}

Keine Interessenkonflikte.

\section{Literatur \\ 1 Schmidt JM: Die philosophischen Vorstel- lungen Samuel Hahnemanns bei der Be- gründung der Homöopathie (bis zum Organon der rationellen Heilkunde, 1810). München, Sonntag, 1990.}

2 Schmidt JM: Is homeopathy a science? Continuity and clash of concepts of science within holistic medicine. J Med Humanit 2009;30:83-97.

3 Schmidt JM: Grundlagen und Entwicklungen in der Homöopathie. Dtsch Med Wochenschr 1993;118:1085-1090.
4 Kiene H: Komplementäre Methodenlehre der klinischen Forschung. Cognition-based Medicine. Berlin, Springer, 2001.

5 Bacon F: Neues Organ der Wissenschaften [1620]. Darmstadt, Wissenschaftliche Buchgesellschaft, 1981. 
6 Hume D: An Enquiry Concerning Human Understanding [1748]. Oxford, Oxford University Press, 1999.

7 Mill JS: System der deduktiven und induk tiven Logik [1843]. Aalen, Scientia, 1968

8 Fisher RA: The Design of Experiments [1935]. Edinburgh, Oliver and Boyd, 1951.

9 Marshall G, et al: Streptomycin treatment of pulmonary tuberculosis. A Medical Research Council investigation. Br Med J 1948;2:769-782.

10 Gold H, Barr DP, DuBois EF, et al. (eds) Cornell Conference on Therapy. Volume One. New York, MacMillan Company, 1946.

11 Beecher HK: The powerful placebo. J Am Med Assoc 1955;17:1602-1606.

12 Kaptchuk TJ: Powerful placebo: the dark side of the randomised controlled trial. Lancet 1998;351:1722-1725.

13 Chalmers I: The Cochrane Collaboration preparing, maintaining, and disseminating systematic reviews of the effects of health care. Ann NY Acad Sci 1993;703: 156-165.

14 Duncker K: Zur Psychologie des produktiven Denkens. Berlin, Springer, 1935.

15 von Weizsäcker V: Warum wird man krank? Ein Lesebuch. Frankfurt/M., Suhrkamp, 2008.

16 Hensel H: Die Simileregel als biologisches Prinzip. Allg Hom Z 1977;222:2-14.

17 von Uexküll $\mathrm{T}$, Wesiak W: Theorie der Humanmedizin. Grundlagen ärztlichen Denkens und Handelns, ed 3. München, Urban \& Schwarzenberg, 1998.

18 Schmidt JM: Thure von Uexkülls semiotisches Modell des Menschen als Grundlage für eine moderne Theorie der Homöopathie. Schweiz Z Ganzheitsmed 2012;24: 233-241.

19 Popper KR: Objektive Erkenntnis. Ein evolutionärer Entwurf. Hamburg, Hoffmann und Campe, 1974.

20 Popper KR: Logik der Forschung [1935]. Berlin, Akademie, 2007.

21 Sackett DL, Richardson WS, Rosenberg W, Haynes RB: Evidence-Based Medicine.
How to Practice and Teach EBM. New York, Churchill Livingstone, 1997.

22 Pocock SJ: Clinical Trials. A Practical Approach. Chichester, Wiley, 1984.

23 Schmidt JM, Kaiser D (Hrsg): Hahnemann S: Gesammelte kleine Schriften. Heidelberg, Haug, 2001.

24 Schmidt JM: Die Publikationen Samuel Hahnemanns. Sudhoffs Archiv 1988;72:1436.

25 Wieland W: Diagnose. Überlegungen zur Medizintheorie. Berlin, de Gruyter, 1975.

26 Wiesing U: Wer heilt, hat Recht? Übe Pragmatik und Pluralität in der Medizin. Stuttgart, Schattauer, 2004

27 Wiesing U: Kunst oder Wissenschaft? Konzeptionen der Medizin in der deutschen Romantik. Stuttgart, FrommannHolzboog, 1995.

28 Wieland W: Verbindlichkeit als wissen schaftstheoretisches Problem?, in Deutsch E, Kleinsorge H, Scheler F (Hrsg): Verbindlichkeit der medizinisch-diagnostischen und therapeutischen Aussage. Stuttgart, Fischer, 1983, pp 35-42.

29 Matthiessen PF: Ärztliche Praxis und wissenschaftlicher Status der Medizin. Forsch Komplementärmed 2006;13:136-139.

30 Schmidt JM: Hahnemanns Homöopathie zwischen rationaler Heilkunde und Heilkunst. Schweiz Z Ganzheitsmed 2011;23: 224-232.

31 Schmidt JM: Homöopathie und Philosophie. Versuch einer philosophischen Rekonstruktion der Begründung der Homöopathie durch Samuel Hahnemann. Scheidewege 1990/91;20:141-165.

32 Ioannides JPA: Why most published research findings are false. PloS Med 2005;2: e124.

33 Schmidt JM: Zur Relevanz wissenschafts-, medizin- und sozialgeschichtlicher Grundlagenforschung. Schweiz Z Ganzheitsmed 2013;25:231-240.

34 Brodbeck KH: Die Herrschaft des Geldes. Geschichte und Systematik, ed 2. Darmstadt, Wissenschaftliche Buchgesellschaft, 2012 .
35 Müller RW: Geld und Geist. Zur Entstehungsgeschichte von Identitätsbewusstsein und Rationalität seit der Antike. Frankfurt/M., Campus, 1977.

36 Graeber D: Schulden. Die ersten 5000 Jahre. Stuttgart, Klett-Cotta, 2012

37 Aristoteles: Politik. Berlin, Akademie, 2011.

38 Schnaas D: Kleine Kulturgeschichte des Geldes. München, Fink, 2010.

39 von Braun C: Der Preis des Geldes. Eine Kulturgeschichte. Berlin, Aufbau, 2012.

40 Mason SF: Geschichte der Naturwissenschaft in der Entwicklung ihrer Denkweisen. Stuttgart, Verlag für Geschichte der Naturwissenschaften und der Technik, 1991.

41 Unschuld PU: Ware Gesundheit. Das Ende der klassischen Medizin. München, Beck, 2009.

42 Unschuld PU: Der Arzt als Fremdling in der Medizin? Standortbestimmung, ed 2. Germering, Zuckschwerdt, 2010.

43 Buschauer W: Homöopathie und Homöopathen. Der Deutsche Apotheker 1988;40: 400-412.

44 Schmidt JM: Taschenatlas Homöopathie in Wort und Bild. Heidelberg, Haug, 2001.

45 Schmidt JM: Believing in order to understand: Hahnemann's hierarchisation of values. Homeopathy 2008;97:156-160.

46 Schmidt JM: Hahnemann's legacy in a new light - a systematic approach to the Organon of Medicine. Homeopathy2005;94:202206.

47 Schmidt JM: Der Simile-Weg als «deuteros plous» in der Arzneitherapie - Konzeption und Rezeption. Documenta Homoeopathica $1992 ; 12: 51-59$.

48 Schmidt JM: Anthropology and medicine. The image of man in different therapeutic approaches. Br Hom J 1993;82:288-296.

49 Tischner R: Das Werden der Homöopathie. Geschichte der Homöopathie vom Altertum bis zur neuesten Zeit. Stuttgart, Hippokrates, 1950. 

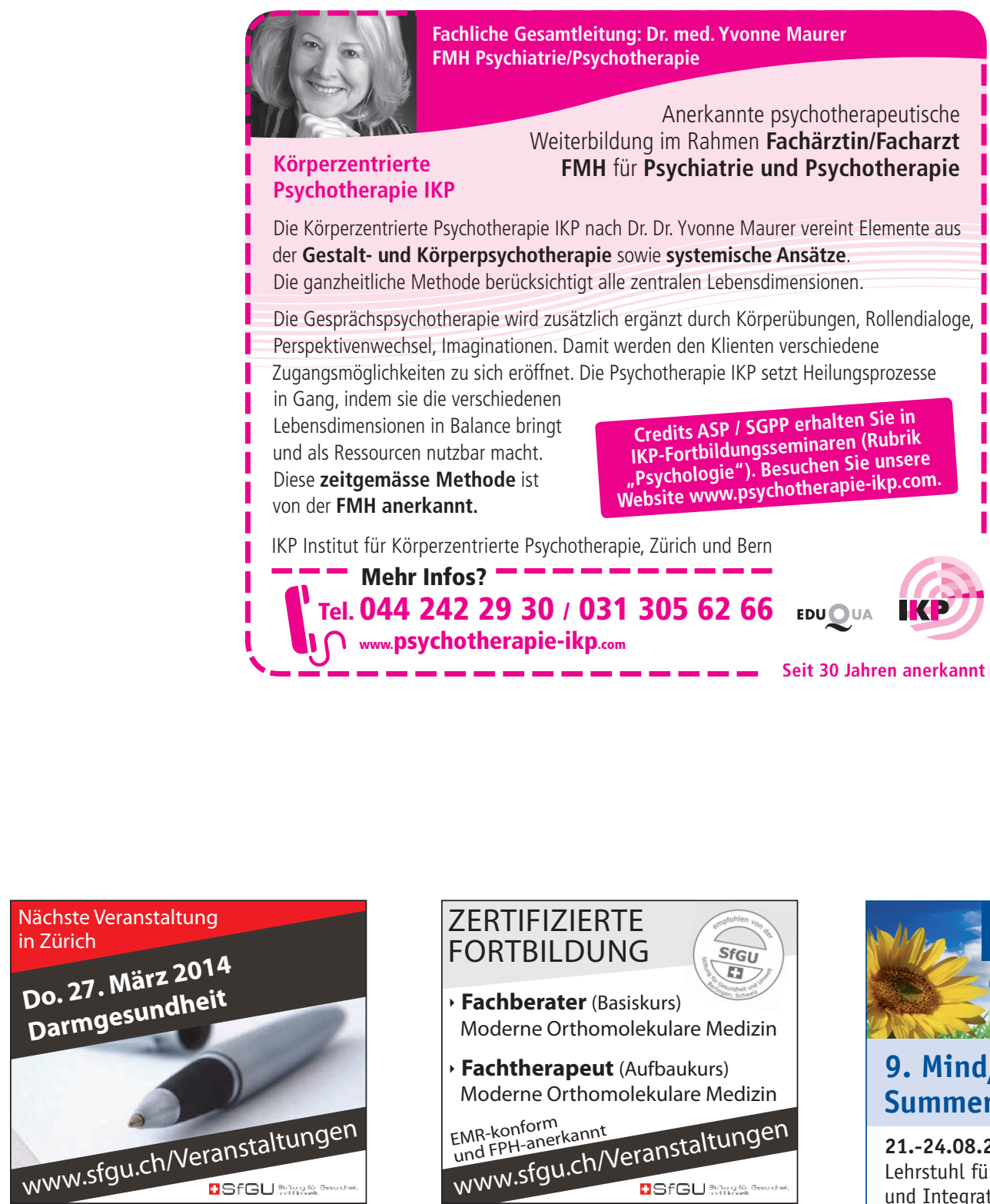

ZERTIFIZIERTE
FORTBILDUNG
- Fachberater (Basiskurs)
Moderne Orthomolekulare Medizin
- Fachtherapeut (Aufbaukurs)
Moderne Orthomolekulare Medizin
EMR-konform
und FPH-anerkannt
WWW.sfgu.ch/Neranstaltungen

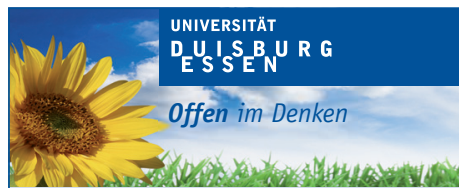

9. Mind/Body Medicine Summer School 2014

21.-24.08.2014|శా 0201-17425512

Lehrstuhl für Naturheilkunde und Integrative Medizin

Jetzt Frühbucherrabatt sichern!

www.mindbodymedicine.de

\section{KARGER}

Hier könnte Ihr Fort- und Weiterbildungsangebot stehen!

Nutzen Sie SCHWEIZERISCHE ZEITSCHRIFT FÜR GANZHEITSMEDIZIN zur effektiven Kommunikation mit Ihrer Zielgruppe! So schaffen Sie die besten Voraussetzungen sowohl gedruckt als auch online gesucht und gefunden zu werden.

Informationen und Angebote bei: Petra Schlegel

Tel. +49 761 45207-13 p.schlegel@karger.com 